\section{BMJ Open Respiratory Research}

\title{
COVID-19 is associated with distinct myopathic features in the diaphragm of critically ill patients
}

Zhonghua Shi, ${ }^{1,2,3}$ Sylvia J P Bogaards, ${ }^{1}$ Stefan Conijn, ${ }^{1}$ Yeszamin Onderwater, ${ }^{1}$ Pedro Espinosa, ${ }^{1}$ Diewertje I Bink, ${ }^{1}$ Marloes van den Berg, ${ }^{1}$ Martijn van de Locht, ${ }^{1}$ Marianna Bugiani, ${ }^{4}$ Hans van der Hoeven, ${ }^{5}$ Reinier A Boon, ${ }^{1}$ Leo Heunks, ${ }^{2}$ Coen A C Ottenheijm ${ }^{1,6}$

\section{ABSTRACT}

Introduction The diaphragm is the main muscle of inspiration, and its dysfunction contributes to adverse clinical outcomes in critically ill patients. We recently reported the infiltration of SARS-CoV-2, and the development of fibrosis, in the diaphragm of critically ill patients with COVID-19. In the current study, we aimed to characterise myofiber structure in the diaphragm of critically ill patients with COVID-19.

Methods Diaphragm muscle specimens were collected during autopsy from patients who died of COVID-19 in three academic medical centres in the Netherlands in April and May $2020(n=27)$. We studied diaphragm myofiber gene expression and structure and compared the findings obtained to those of deceased critically ill patients without COVID-19

$(n=10)$.

Results Myofibers of critically ill patients with COVID-19 showed on average larger crosssectional area (slow-twitch myofibers: $2441 \pm 229$ vs $1571 \pm 309 \mu \mathrm{m}^{2}$; fast-twitch myofibers: $1966 \pm 209$ vs $1225 \pm 222 \mu \mathrm{m}^{2}$ ). Four critically ill patients with COVID-19 showed extremely large myofibers, which were splitting and contained many centralised nuclei. RNA-sequencing data revealed differentially expressed genes involved in muscle regeneration.

Conclusion Diaphragm of critically ill patients with COVID-19 has distinct myopathic features compared with critically ill patients without COVID-19, which may contribute to the ongoing dyspnoea and fatigue in the patients surviving COVID-19 infection.

\section{(c) Author(s) (or their} employer(s)) 2021. Re-use permitted under CC BY-NC. No commercial re-use. See rights and permissions. Published by BMJ

For numbered affiliations see end of article.

Correspondence to Dr Coen A C Ottenheijm; c.ottenheijm@ amsterdamumc.nl

\section{INTRODUCTION}

In critically ill patients, diaphragm weakness may develop and contribute to adverse clinical outcomes including ventilator weaning failure, prolonged hospitalisation and mortality. ${ }^{1}$ Diaphragm weakness in these patients is characterised by pathological features in myofibers, including reduced cross-sectional area $(\mathrm{CSA})$, that is,
Key messages

\section{What is the key question?}

- RNA-sequencing results from the diaphragm of deceased patients with COVID-19 suggest the activation of gene pathways involved in myofiber regeneration.

\section{What is the bottom line?}

In line with the RNA-sequencing results, the results of structural analyses of the diaphragm specimens show enlarged and splitting myofibers with central nuclei.

\section{What read on?}

- The diaphragm of deceased patients with COVID-19 displays distinct pathological changes, which may contribute to the ongoing dyspnoea and fatigue in patients who survive COVID-19.

atrophy. ${ }^{2-4}$ Recently, we reported evidence for infiltration of SARS-CoV-2 in diaphragm myofibers. ${ }^{5}$ We also demonstrated increased expression of genes involved in fibrosis and histological evidence for the development of fibrosis in the diaphragm of critically ill patients with COVID-19 compared with the diaphragm of critically ill patients without COVID-19. ${ }^{5}$ In the current study, we characterised myofiber structure in the diaphragm of critically ill patients with COVID-19. Myofiber structure is an important determinant of diaphragm contractility. Consequently, impaired myofiber structure might contribute to diaphragm muscle weakness, and, thereby, impact weaning outcome and contribute to the persistent dyspnoea and fatigue reported in post-COVID-19 patients. ${ }^{6}$ Thus, we aimed to study the structure of diaphragm myofibers in critically ill patients with COVID-19 and compared the findings to those of patients without COVID-19. 


\section{METHODS}

\section{Patients}

Diaphragm specimens of critically ill patients with COVID-19 (COVID-19-intensive care unit (ICU), $\mathrm{n}=27$ ) and of critically ill patients without COVID-19
(control-ICU, $\mathrm{n}=10$ ) were collected at autopsy. Diagnosis of COVID-19 was based on SARS-CoV-2 detection by real-time quantitative PCR, in nasopharyngeal swabs. Samples were obtained from the middle of the left costal diaphragm (figure 1A), 16-72 hours

A Schematic of the location of biopsy (bottom view)

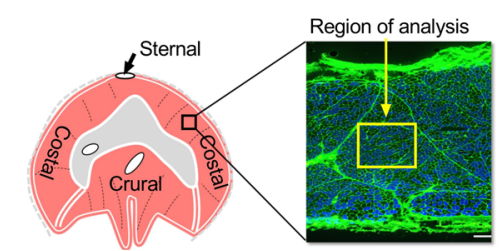

B Volcano plot

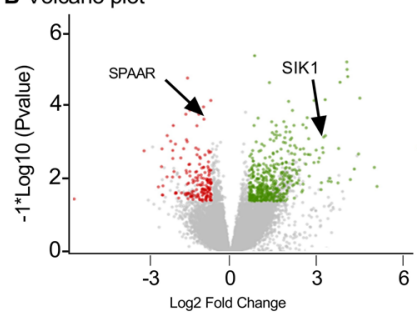

C Gene ontology

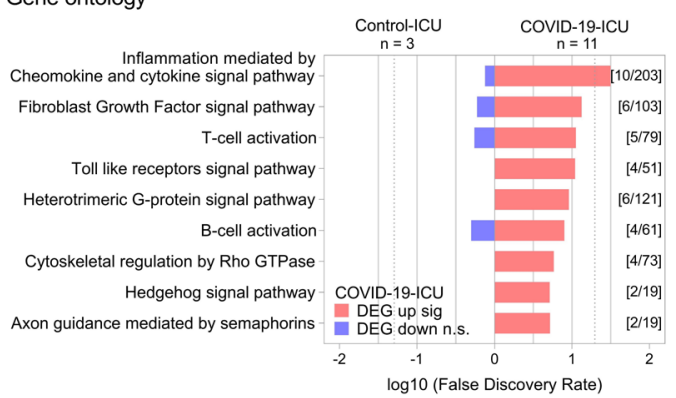

COVID-19-ICU upregulated Not significantly regulated - COVID-19-ICU downregulated

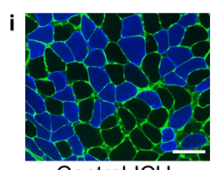

Control-ICU

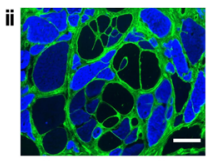

COVID-19-ICU
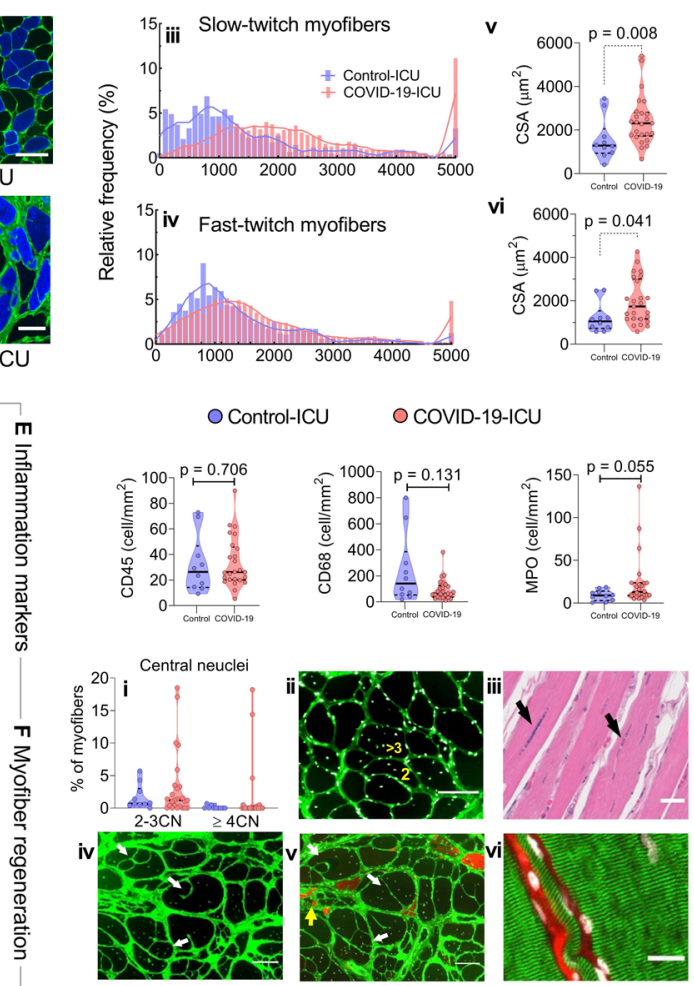

Figure 1 (A)Schematic of the location of the biopsy obtained (balck and yellow squares). (B) Volcano plot showing the 315 upregulated and 281 downregulated genes (SPAAR: small regulatory polypeptide of amino acid response; SIK1: salt inducible kinase) and (C) Gene ontology biological process enrichment analysis (PANTHER) showing upregulated/downregulated processes in the diaphragm of COVID-19-ICU $(n=11)$ patients compared with Control-ICU ( $n=3)$. (DEG: differentially expressed gene). (D) Cross-sectional area (CSA) in COVID-19-ICU ( $n=25)$ and Control-ICU patients $(n=10)$. Representative images of one patient from Control-ICU (i) and COVID-19-ICU (ii), respectively. Cross-sections stained for fast myosin heavy chain isoforms (blue) to identify fast-twitch myofibers; slow-twitch myofibers are black; in green WGA to demarcate myofibers; bar $=100 \mu \mathrm{m}$. Note the extremely large myofibers in the COVID-19-ICU patient (patient \#28 is shown). Distribution of CSA of slow-twitch (iii) and fast-twitch (iv) myofibers of COVID-19-ICU patients (red) skewed towards larger CSA compared with Control-ICU patients (purple). The CSA of both the slow-twitch and fast-twitch myofibers were significantly larger in COVID-19-ICU patients compared with Control-ICU patients (v, vi). Data are visualised using histogram and violin plot. Each dot represents mean of the CSA from an individual patient. P values were obtained from linear mixed model analysis. (E) Quantification of CD68 + macrophages, CD45 + leucocytes, and MPO+ neutrophils in COVID-19-ICU and Control-ICU patients. No differences were observed between groups. The data are visualised using violin plot with each dot representing the value of an individual patient. P values were obtained from Mann-Whitney $U$ test. (F) Signs of myofiber regeneration in COVID-19-ICU patients. (i) Quantification of the number of central nuclei in cross-sectioned myofibers. Note that four COVID19-ICU patients (\#2;3;28;31) had an increased number of myofibers with 2-3 central nuclei; three of these four patients had myofibers with more than three central nuclei (up to 18 per myofiber). (ii) Cross-section of patient \#31 stained with WGA and DAPI to localise the myonuclei; yellow numbers indicate representative myofibers with multiple central nuclei; bar=100 $\mu \mathrm{m}$. (iii) H\&E staining (formalin fixed sample) of a longitudinal section of patient \#3 showing multiple 'trains' of central nuclei (arrows); bar $=100 \mu \mathrm{m}$. (iv, v). Two serial cross-sections (separated by $100 \mu \mathrm{m}$ ) of patient \#28, showing splitting of myofibers; arrows indicate the same myofibers with splitting events; in green WGA to demarcate myofibers. Also, note the large variation of myofiber CSA in this patient. In (v), $\alpha-\mathrm{MHC} 3$ antibody staining (red) visualises myofibers expressing an embryonic isoform of myosin heavy chain. Note that the split fibers are adult, non-regenerating myofibers (white arrows) and that the tiny myofibers surrounded by fibrosis are regenerating (yellow arrows); bar=100 $\mu \mathrm{m}$. (vi) Longitudinal section of patient \#28 stained with WGA to demarcate the membrane (red) and DAPI to localise the myonuclei (white); the $\alpha$-actinin antibody staining in green shows the Z-disc organisation. From left to the right, the diameters for the fibers are 40, 10 and $160 \mu \mathrm{m}$, respectively; bar $=20 \mu \mathrm{m}$. ICU, intensive care unit; WGA, wheat germ agglutinin; DAPI, 4',6-diamidino-2-phenylindole; MHC, myosin heavy chain. 
postmortem. Patients were recruited in three medical centres in the Netherlands. Clinical data were collected retrospectively.

\section{Transcriptome sequencing (RNA-seq)}

RNA and library preparation integrity were confirmed with a LabChip Gx Touch 24 (Perkin Elmer). The function of genes was referred to the 'National Center for Biotechnology Information (NCBI)'s Gene' and 'GeneCards' databases. Because only samples of sufficient integrity were further processed for RNA sequencing, 3 control-ICU patients and 11 COVID-19-ICU patients were analysed.

\section{Histology and immunohistochemistry to study myofiber structure}

Serial cryosections $(8 \mu \mathrm{m}$ thick) were cut from the frozen biopsies (perpendicular to diaphragm fiber direction) and stained to study (1) myofiber CSA using wheat germ agglutinin staining and myofiber type using myosin heavy chain antibodies, (2) myofiber nuclei using 4',6-diamidino-2-phenylindole staining and (3) inflammatory infiltrates using CD45, CD68 and myeloperoxidase (MPO) antibodies. To study the striation pattern, longitudinal sections were cut and studied by $\alpha$-acting staining (to visualise the $z$-disc of sarcomeres) and by H\&E staining (to visualise the overall structure of the myofibers in formalin-fixed tissue). Myofiber CSA was obtained from \pm 100 myofibers per type per biopsy (analysis of $>100$ myofibers did not affect the coefficient of variation $(\mathrm{CV})$ of the CSA). Inflammatory cell counts (leucocytes, macrophages and neutrophils) were obtained from three random selected viewing fields per section per biopsy $\left(0.5 \mathrm{~mm}^{2}\right.$ for fibrosis and $1 \mathrm{~mm}^{2}$ for inflammatory cells, respectively). Central nucleus was defined as the presence of one nucleus anywhere in the sarcoplasm and at least $5 \mu \mathrm{m}$ inside the sarcolemma.

\section{Statistical analysis}

Data were analysed with IBM SPSS Statistics V.22 (IBM) and visualised with GraphPad Prism V.7.0 (GraphPad Software, USA). For repeated measurements (ie, CSA and inflammatory cells), CV was calculated to assess the variations in individual patients. Linear mixed model was applied with patients as the random factor and group as fixed factor to compare the difference between two groups of patients. Assumption of normality was tested by the Shapiro-Wilk normality test. Log transformation was performed if necessary. For non-repeated measurements, the Mann-Whitney U test or Student's t-test was applied for quantitative variables. A $\chi^{2}$ or Fisher's exact test was used to compare qualitative variables. Data were expressed as mean $( \pm \mathrm{SEM})$, median $(\mathrm{IQR})$ or frequencies and percentage, as indicated. We used a two-sided significance level of $5 \%$ for all analyses.

\section{Patient and public involvement}

Patients or the public were not involved in the design, conduct, reporting and dissemination plans of our research.

\section{RESULTS}

Between both groups, there were no significant differences in the number of days on mechanical ventilation, the length of ICU stay, and the administration of neuromuscular blocking agents and steroids. None of the patients had pre-existing neuromuscular disease or chronic cardiopulmonary disease. All patients with COVID-19 were admitted to the ICU because of COVID-19 infection and required invasive mechanical ventilation. More clinical characteristics are in table 1 and online supplemental table 1.

First, we investigated gene expression in diaphragm tissue from eleven COVID-19-ICU patients and three control-ICU patients by RNA sequencing (only samples with RNA of sufficient integrity were used). A total of 596

\begin{tabular}{llll}
\hline \multicolumn{2}{l}{ Table 1 Summary of the demographic and clinical characteristics } & & \\
\hline Characteristic & COVID-19-ICU (n=27) & Control-ICU (n=10) & P value \\
\hline Age, year & $71(61-74)$ & $67(64-73)$ & 0.827 \\
Sex, male (\%) & $21(78)$ & $6(60)$ & 0.248 \\
\hline BMI, kg/m ${ }^{2}$ & $27(26-31)$ & $24(21-28)$ & 0.014 \\
Duration ICU stay, days & $13(8-27)$ & $11(3.3-12.3)$ & 0.113 \\
\hline Duration of IMV, days & $13(6-27)$ & $9(1-12)$ & 0.053 \\
\hline Duration of NMB administration, hours & $0(0-98)$ & $0(0-240)$ & 0.821 \\
\hline Systemic steroid administration, $\mathrm{n}(\%)$ & $12(46)$ & $7(70)$ & 0.182 \\
\hline Maximal CRP, mg/L & $310(260-383)$ & $312(135-481)$ & 0.969 \\
\hline
\end{tabular}

Data are expressed as median (IQR) or percentage (n, (\%). P values were obtained from Mann-Whitney $U$ test or $\chi^{2}$ test. Steroid administration was defined as more than $10 \mathrm{mg}$ for $>2$ days.

$\mathrm{BMI}$, body mass index; CRP, C reactive protein; ICU, intensive care unit; IMV, invasive mechanical ventilation; NMB, neuromuscular blocking agents. 
(315 upregulated; 281 downregulated) of 27647 detected genes were differentially expressed (fold-change $> \pm 1.5$; $\mathrm{p}<0.05$; figure 1B). Among the five most upregulated and downregulated genes were the small regulatory polypeptide of amino acid response (SPAAR, downregulated) and the salt inducible kinase (SIK1, upregulated), both expressed in the direction of promoting muscle regeneration. ${ }^{78}$ However, genes that are known to be involved in critical illness-associated muscle atrophy, such as the ubiquitin-proteasome and autophagy-lysosome pathways, ${ }^{9}$ were not differentially expressed between groups. Gene ontology analysis by protein analysis through evolutionary relationships (PANTHER) revealed upregulation of the Fibroblast Growth Factor pathway (as reported before, ${ }^{5}$ and of several pathways involved in inflammation (figure 1C).

Prompted by the gene expression analyses, diaphragm sections were stained to study myofiber CSA, inflammatory infiltrates, localisation of myonuclei and the striation pattern of the myofibers. The distribution of myofiber CSA in COVID-19-ICU patients shifted towards larger myofibers compared with control-ICU patients (figure 1D). The average myofiber CSA was larger in COVID-19-ICU compared with control-ICU patients (slow-twitch: $2441 \pm 229$ vs $1571 \pm 309 \mu^{2}$, respectively, $p=0.008$; figure $1 \mathrm{Diii} / \mathrm{v}$; fast-twitch: $1966 \pm 209$ vs . $1225 \pm 222 \mu^{2}$, respectively, $\mathrm{p}=0.041$; figure $1 \mathrm{Div} / \mathrm{vi}$; note that analysis of minimal Feret diameter rendered comparable results. Data are shown in the online supplement, online supplemental figure 1). In COVID-19-ICU patients, the maximum CV was $140 \%$ for slow-twitch myofiber and $132 \%$ for fasttwitch myofibers. The average CV, however, was comparable between COVID-19-ICU and control-ICU patients (slow-twitch: $49 \pm 21 \%$ vs $51 \pm 12 \%$, respectively, $\mathrm{p}=0.46$; fast-twitch: $50 \pm 23 \%$ vs $48 \pm 12 \%$, respectively, $\mathrm{p}=0.653$ ). No significant differences were observed between groups for the number of lymphocytes $\left(\mathrm{CD} 45^{+}\right)$, macrophages $\left(\mathrm{CD}^{+} 8^{+}\right)$and neutrophilic granulocytes $\left(\mathrm{MPO}^{+}\right)$ (figure 1E).

In four COVID-19-ICU patients (mechanical ventilation duration 5-49 day), we observed diaphragm myofibers with central nuclei (figure 1Fi-iii), indicative of myofiber regeneration. Longitudinal sections showed that, occasionally, these central nuclei were organised in 'trains' (figure 1Fiii), as frequently observed in muscular dystrophies. These four patients also displayed extremely large myofibers (CSA $>10000 \mu^{2}$ ) and splitting of these large myofibers (figure 1Fiv-v), consistent with diaphragm myopathy and regeneration, which was not present in control-ICU patients. Significant higher variation in myofiber CSA was observed in these four patients compared with control-ICU patients (CV: slowtwitch: $81 \% \pm 40 \%$; fast-twitch: $90 \% \pm 32 \%$ ). Importantly, longitudinal sections of the diaphragms showed clear striation patterns across these extremely large myofibers, indicating increased myofibrillar content rather than the accumulation of organelles such as mitochondria or of degradation products resulting from postmortem autolysis (figure 1Fvi).

\section{DISCUSSION}

The present study reveals distinct pathological changes in the diaphragm myofibers of critically ill patients with COVID-19. A striking observation was the larger CSA of myofibers, which was independent of the duration of mechanical ventilation (table 1). It is likely that the increased variation of myofiber CSA reflects myofiber regeneration and splitting, as supported by the central nuclei in myofibers and the RNA sequencing data showing DEGs involved in myofiber regeneration. Our previous work on these diaphragm samples showed increased fibrosis, ${ }^{5}$ which suggests that the myofiber regeneration was-at least partly-pathological. We speculate that these pathological changes may impact the contractility of the diaphragm in patients with COVID-19, and contribute to the persisting sensation of dyspnoea and fatigue that a subset of patients experience. ${ }^{6}$ Myofiber regeneration and splitting occurs in response to myofiber injury. The aetiology of myofiber injury in COVID-19-ICU patients is unclear. It is tempting to speculate that the pathological changes might be a direct effect of the virus, as we have recently shown that SARS-CoV-2 particles may infiltrate diaphragm myofibers, ${ }^{5}$ but it remains to be established whether this affects gene expression. Alternatively, it may be explained by a high respiratory drive in mechanically ventilated patients with COVID- $19,{ }^{10}$ as high respiratory loading is known to cause diaphragm myofiber injury. ${ }^{11}$ However, this was not specifically studied in our patients.

This study has some limitations. First, without non-critically ill patients as a control group, it is unclear whether myofibers of COVID-19-ICU patients are less atrophic than those of control-ICU patients or are more hypertrophic compared with non-critically ill patients. Furthermore, as the current study was cross-sectional of nature, we cannot exclude that baseline variations affected the study outcomes. Importantly, none of the patients had a history of neuromuscular diseases, which reduces the possibility of pre-existing diaphragm pathology. Second, the number of patients recruited was small, particularly for the control-ICU patients $(n=10)$. Consequently, although groups were comparable regarding the duration of mechanical ventilation and the use of corticosteroids, both risk factors for diaphragm pathology, ${ }^{1}$ they were not for body mass index. Additionally, although proposed as a risk factor for diaphragm pathology, ${ }^{1}$ sepsis was present in only three of the control-ICU patients (and in none of the COVID-19-ICU patients), thus, it is unlikely that this contributed to the diaphragm pathology observed in the current study. Furthermore, some of the pathological changes were observed in a subset of COVID-19-ICU patients. However, we have published several studies ${ }^{2-4}$ that included critically ill patients undergoing elective surgery and not in a single case did we observe the pathological changes (increased variation of myofiber CSA, extremely large myofiber CSA, central nuclei, myofiber splitting) reported here. 
Nevertheless, caution is warranted when drawing conclusions based on these findings. They should be considered as hypothesis generating, and future studies should focus on the underlying mechanisms and risk factors for these myopathic features in the diaphragm. Third, in both groups the samples were collected 16-72hours postmortem, and tissue degradation may have occurred. However, the longitudinal sections revealed preserved striation pattern (figure 1Fvi), indicating that gross myofiber morphology was intact. Finally, only 3 of the 10 control-ICU patients had a viral infection, which warrants caution when comparing direct effects of SARS-CoV-2 with those of other viruses. Furthermore, RNAsequencing analyses were performed in a subset of patients, therefore, caution is warranted when relating the findings from the RNA-sequencing analyses to those from the histological analyses.

\section{CONCLUSION}

In summary, the diaphragm of deceased COVID-19 patients shows distinct myopathic changes. Although it is unknown whether these changes in the diaphragm persist in COVID-19 survivors, it is tempting to speculate that they contribute to the ongoing dyspnoea and fatigue in those patients.

\section{Author affiliations}

'Department of Physiology, Amsterdam UMC Locatie VUmc, Amsterdam, The Netherlands

${ }^{2}$ Department of Intensive Care Medicine, Amsterdam UMC Locatie VUmc, Amsterdam, The Netherlands

${ }^{3}$ Department of Intensive Care Medicine, Beijing Tiantan Hospital, Capital Medical University, Beijing, People's Republic of China

${ }^{4}$ Department of Pathology, Amsterdam UMC Locatie VUmc, Amsterdam, The Netherlands

${ }^{5}$ Department of Intensive Care Medicine, Radboudumc, Nijmegen, The Netherlands

${ }^{6}$ Department of Cellular and Molecular Medicine, University of Arizona, Tucson, Arizona, USA

Contributors CACO, LH, ZS and HvdH contributed to conception and design. ZS, SJPB, SC, YO, PE, DIB, MB, MvdL, MvdB and RAB contributed to acquisition and analysis of data. ZS, SJPB, MvdB, MvdL, RAB, CACO and LH contributed to interpretation of data. ZS, CACO, LH and HvdH contributed to drafting the manuscript. All authors reviewed, edited and approved the final manuscript.

Funding Research reported in this work was supported by a grant from the National Institutes of Health-Heart Lung and Blood Institute R01HL121500 (CACO).

Competing interests $\mathrm{LH}$ reports grants and personal fees from Liberate medical, personal fees from Getinge, personal fees from Fisher and Paykel, outside the submitted work.
Patient consent for publication Not required.

Ethics approval The study was approved by the medical ethical committee at Amsterdam UMC (\#2020-167).

Provenance and peer review Not commissioned; internally peer reviewed.

Data availability statement Data are available on reasonable request. All data relevant to the study are included in the article or uploaded as online supplemental information.

Supplemental material This content has been supplied by the author(s). It has not been vetted by BMJ Publishing Group Limited (BMJ) and may not have been peer-reviewed. Any opinions or recommendations discussed are solely those of the author(s) and are not endorsed by BMJ. BMJ disclaims all liability and responsibility arising from any reliance placed on the content. Where the content includes any translated material, BMJ does not warrant the accuracy and reliability of the translations (including but not limited to local regulations, clinical guidelines, terminology, drug names and drug dosages), and is not responsible for any error and/or omissions arising from translation and adaptation or otherwise.

Open access This is an open access article distributed in accordance with the Creative Commons Attribution Non Commercial (CC BY-NC 4.0) license, which permits others to distribute, remix, adapt, build upon this work non-commercially, and license their derivative works on different terms, provided the original work is properly cited, appropriate credit is given, any changes made indicated, and the use is non-commercial. See: http://creativecommons.org/licenses/by-nc/4.0/.

\section{REFERENCES}

1 Dres M, Goligher EC, Heunks LMA, et al. Critical illness-associated diaphragm weakness. Intensive Care Med 2017;43:1441-52.

2 Hooijman PE, Beishuizen A, de Waard MC, et al. Diaphragm fiber strength is reduced in critically ill patients and restored by a troponin activator. Am J Respir Crit Care Med 2014;189:863-5.

3 Lindqvist J, van den Berg M, van der Pijl R, et al. Positive endexpiratory pressure ventilation induces longitudinal atrophy in diaphragm fibers. Am J Respir Crit Care Med 2018;198:472-85.

4 van den Berg M, Hooijman PE, Beishuizen A, et al. Diaphragm atrophy and weakness in the absence of mitochondrial dysfunction in the critically ill. Am J Respir Crit Care Med 2017;196:1544-58.

5 Shi Z, de Vries HJ, Vlaar APJ, et al. Diaphragm pathology in critically ill patients with COVID-19 and postmortem findings from 3 medical centers. JAMA Intern Med 2021;181:122-4.

6 Chen T, Wu D, Chen H, et al. Clinical characteristics of 113 deceased patients with coronavirus disease 2019: retrospective study. BMJ 2020;368:m1091.

7 Berdeaux R, Goebel N, Banaszynski L, et al. SIK1 is a class II HDAC kinase that promotes survival of skeletal myocytes. Nat Med 2007;13:597-603.

8 Matsumoto A, Pasut A, Matsumoto M, et al. Mtorc1 and muscle regeneration are regulated by the LINC00961-encoded SPAR polypeptide. Nature 2017;541:228-32.

9 Llano-Diez M, Fury W, Okamoto $\mathrm{H}$, et al. RNA-sequencing reveals altered skeletal muscle contraction, E3 ligases, autophagy, apoptosis, and chaperone expression in patients with critical illness myopathy. Skelet Muscle 2019;9:9.

10 Esnault P, Cardinale M, Hraiech S, et al. High respiratory drive and excessive respiratory efforts predict relapse of respiratory failure in critically ill patients with COVID-19. Am J Respir Crit Care Med 2020;202:1173-8.

11 Reid WD, Belcastro AN. Time course of diaphragm injury and calpain activity during resistive loading. Am J Respir Crit Care Med 2000;162:1801-6. 